Pascale Hadermann

Universiteit Gent \& Gramm-R

Michel Pierrard

Vrije Universiteit Brussel \& Gramm-R

\title{
La construction corrélative et les marqueurs en qu-
}

\section{INTRODUCTION}

Face à la dichotomie bancale « coordination-subordination » rappelée en ouverture de ce numéro, certains linguistes, dans leurs descriptions de la connexion de prédications en français, tendent à récupérer le mode syntaxique de la corrélation, disparu avec l'instauration de la grammaire scolaire (Chervel 1977 ; Mignon 2009), sans s'interroger sur la pertinence de cette récupération dans une langue a priori considérée, d'un point de vue typologique, comme ne possédant pas de structures corrélatives (cf. Lipták 2009). En effet, pour la famille indoeuropéenne, les corrélatives ne seraient attestées qu'en latin, en ancien anglais, en hittite, en lycien, en russe médiéval, en bulgare, en macédonien, en serbocroate et en polonais. Toutefois, il a été démontré que le schéma corrélatif, dont nous présenterons les caractéristiques $\S 2$, se retrouve aussi dans des structures à première vue différentes, telles que les « comparatives corrélatives » de type plus tu lis, moins tu comprends, qui apparaissent dans des langues dites " non corrélatives » (Lipták, 2009 : 18). Le rapprochement s'appuie sur le fait que dans des langues comme le hindi - qui exploite le schéma corrélatif prototypique - la corrélative et la " comparative corrélative » se construisent de la même manière. Ce parallélisme amène des auteurs, tels que M. Den Dikken (2005), à creuser le fonctionnement d'énoncés qui expriment une comparaison entre deux prédications lire et comprendre de l'exemple supra - dans des langues "non corrélatives ", comme l'anglais, l'allemand, le néerlandais, etc. et à les relier effectivement au schéma corrélatif.

À la suite de ce double constat - insuffisance de l'opposition dichotomique " coordination/subordination » d'une part, importance de la corrélation pour la description des modes de liaison de prédications d'un point de vue typologique d'autre part - notre contribution se donne pour objectif d'examiner, en s'inspirant entre autres de l'exemple du latin, si la corrélation permet de mieux 
appréhender le fonctionnement des marqueurs en $q u$ - du français moderne. Ceux-ci sont généralement considérés comme des icones de la subordination ${ }^{1}$ (cf. 1a-e), mais certains emplois paraissent déviants (cf. 2a-d) :

(1) a. Il a vu l'homme qui a tué le policier / que tu as dénoncé à la police.

b. Il dit en souriant quelque chose à quoi évidemment je ne répondis pas. (Lefeuvre \& Rossari, $2008:$ 87)

c. Je vous montrerai la ville où je suis né.

d. Il m'a téléphoné quand il est arrivé en France.

e. Comme il pleuvait, j'ai pris un parapluie.

(2) a. Sous l'assaut, Venant ne broncha pas d'un cil. Ce qui plut infiniment à Didace. (Guèvrement, Le survenant, 1974)

b. Oublier ces évidences, c'est se priver des clés essentielles de compréhension des réalités actuelles. D'où l'utilité du rapide cours d'histoire dispensé par [...]. (Le Monde, 01.08.1989)

c. Mais celui qui a dit cela, celui-là sera maudit jusqu'à la fin des temps.

d. Quand tu seras plus grand, alors tu comprendras.

Les marqueurs en $q u$ - dans (2a-b) introduisent des énoncés non intégrés, ce qui est souligné par la ponctuation. Dans $(2 \mathrm{c}-\mathrm{d})$, la forme en $q u$ - est reprise par un morphème coréférentiel, ce qui semble remettre en question son rôle de subordonnant.

Avant d'examiner les divers emplois des marqueurs en $q u-$, nous rappellerons d'abord les caractéristiques de la corrélation dans des langues classiques comme le latin $(\S 2)$. Ensuite, nous examinerons dans quelle mesure ces caractéristiques peuvent aider à mieux comprendre le fonctionnement des marqueurs en $q u$ - en jonction interpropositionnelle ( $\S 3$ ). Pour ce faire, nous dresserons un tableau aussi complet que possible des différents emplois, ce qui nous permettra d'isoler les propriétés du marqueur dans chaque tour (§ 4-7). Les emplois seront présentés indépendamment du registre dont ils proviennent ou de la fréquence des occurrences ${ }^{2}$, dans la mesure où le but de la présente contribution est de décrire la gamme potentielle des configurations possibles des marqueurs en qu-. Une étude variationnelle et des fréquences sur corpus serait certes intéressante, mais dépasse les objectifs de cette contribution.

1. Les marqueurs suivants seront étudiés : qui, quoi/que, quand, où et comme(nt). Nous n'approfondirons pas le fonctionnement de dont et de combien. L'argumentation s'étend en principe aussi aux emplois « conjonctifs » de que : Il sait que je viendrai. Pour des raisons de place, ces questions ne seront pas traitées dans cette contribution.

2. Nos données proviennent de la consultation d'ouvrages de référence, de bases de données comme Frantext et les Archives du Monde, ainsi que d'Internet. Les exemples qui ne sont pas accompagnés de références sont construits pour les besoins de la démonstration. 


\section{LA CORRÉLATION}

La corrélation telle qu'elle apparait dans les langues dites " corrélatives » se caractérise avant tout par la présence de deux morphèmes, souvent apparentés, qui introduisent chacun une des deux parties de l'énoncé « corrélatif ». Cette présence simultanée de deux marqueurs a amené A. Minard (1936, cité par Mellet, 2013 : 87), dans sa description du sanscrit, à parler de structures à « diptyque normal » : pour les langues classiques indo-européennes, il s'agit plus spécifiquement de séquences où le premier élément est une forme relative et où le second élément est un morphème anaphorique ou « résomptif ».

(3)

$\begin{array}{llllll}\text { Quam quisque norit } & \text { artem, in hac } & \text { se exerceat } \\ \text { Rel.sgf.acc chacun.sgm connâtre.pres.s.3S } & \text { art.sg.acc } & \text { dans } & \text { dém.sgf.abl refl.acc exercer.subj.s3S } \\ \text { "Lequel art chacun connait, qu'il s'exerce dans celui-là. " (Creissels, 2006:247) } & \end{array}$

Formellement, la corrélation est une structure avec une relative à antécédent interne qui se trouve extraposée par rapport à la seconde proposition. Il arrive que l'antécédent ne soit pas matériellement présent :

(4)

quem uides, eum ignoras : illum nominas, quem non uides

diptyque normal diptyque inverse

“Celui que tu vois, tu l'ignores; et tu nommes celui que tu ne vois pas " (Plaute, cité par Fruyt, 2005: 28)

La seconde proposition se caractérise par la présence d'un morphème démonstratif en position initiale. Les morphèmes relatif et démonstratif exercent chacun une fonction au sein de la proposition qu'ils introduisent et le relatif est coréférentiel du démonstratif qui, à son tour, renvoie anaphoriquement au relatif : la première proposition ne se comprend que lorsque la seconde est énoncée et la seconde a besoin de la première pour être comprise. Cette double référence ana-cataphorique se trouve à la base du rapport syntaxique d'interdépendance qui se dégage de la structure : les deux propositions entretiennent une relation $\mathrm{d}^{\prime}$ « ordination » bilatérale ou équipotente (Saez, $2011: 56$ ), plutôt qu'un rapport de subordination.

Outre les diptyques normaux en "relatif - démonstratif », il y a lieu de distinguer les diptyques inverses en « démonstratif - relatif » (Tam ego homo sum quam tu, 'je suis autant homme que toi', Mellet, 2013 : 94). Cette constellation morphologique inversée affecte avant tout le fonctionnement pragmatique (effet de mise en relief selon Fruyt 2005), alors que le fonctionnement syntaxique et sémantique reste bien celui d'un énoncé corrélatif : syntaxiquement, nous avons affaire à la saturation d'une fonction au sein de chaque proposition et à l'établissement d'une double ligature ; sémantiquement, par le biais du rapport ana-cataphorique, il se crée " une opération de fléchage » avec le démonstratif, opération qui, grâce à la présence du morphème $q u-$, sera accompagnée d'un parcours de variables (cf. Mellet, 2013 : 90). 
D'après les observations de la linguistique typologique, la corrélation à diptyque normal est très bien attestée dans les langues indo-européennes anciennes, mais n'aurait pas ou peu survécu en français, qui semble privilégier le diptyque inverse, un système à plus forte intégration, menant à une dépendance unilatérale au lieu d'une interdépendance (cf. Srivastav 1991 ; Lipták 2009).

\section{LES MARQUEURS EN QU-, PROFORMES INDÉFINIES ET APPEL DE SPÉCIFICATION}

Les différentes grilles proposées pour analyser le fonctionnement d'un marqueur confondent souvent les caractéristiques du mode de connexion et celles du marqueur (cf. par ex. Grevisse \& Goosse, 1993 : 1604 ; Wilmet, 2010 : 352361) dans la mesure où elles partent généralement du présupposé non explicité « qu'il doit y avoir une régularité dans l'interface entre les composantes morphologique et syntaxique de la description » (Deulofeu, $2008: 35$; cf. aussi Corminboeuf, 2007 : 178). Dans cette logique, le relatif, par exemple, instaure par définition un rapport de subordination entre propositions. Cette interface pose toutefois de nombreux problèmes car les marqueurs en $q u$ - présentent une grande flexibilité et variabilité dans leur mode de connexion (cf. e.a. Deulofeu 2008 ; Corminboeuf 2007 ; Benzitoun 2008 ; etc.). L'identification complète d'un marqueur devra donc nécessairement passer par la mise en évidence (i) de ses caractéristiques morpho-sémantiques propres, ses propriétés internes et (ii) de ses propriétés externes, i.e. son implication envers les deux propositions (ou prédications, terme plus large que nous utiliserons dorénavant) qu'il lie (cf. Pierrard 2013).

Du point de vue de leurs propriétés internes, les marqueurs en $q u$ - du français (qui, que/quoi, où, quand et comme/comment/combien) sont « originellement et fondamentalement des indéfinis » (Le Goffic, $1993: 40$ ). L'indéfinition n'est cependant pas la seule propriété interne des marqueurs en $q u-$; ils se caractérisent aussi, à cause de cette spécification interne restreinte, par le trait général [+ appel de spécification], ce qui amène M. Pierrard (1998) à les définir comme des « proformes indéfinies sous-spécifiées en recherche de spécification » (cf. aussi Lefeuvre \& Pierrard 2008 et Pierrard 2005) :

La forme qu- [...] délimite un champ où doit s'inscrire la référence : pour qu-, il s'agit des champs de l'animé, de l'inanimé, de l'espace, du temps, de la propriété, sous leur forme la plus générale et prototypique à la fois. L'extension de la proforme n'étant pas déterminable en soi, l'emploi en tant qu'item lexical pur est devenu impossible. L'exploitation de la proforme essentielle indéfinie entraînera nécessairement une spécification du champ abordé par des apports contextuels complémentaires. (Pierrard, $1998: 37)$ 
Cette recherche d'un apport de spécification va orienter les propriétés externes des proformes indéfinies. L'appel de spécification débouche, en effet, sur plusieurs propriétés externes qui permettent d'affiner le fonctionnement des marqueurs en $q u-$ :

- la recherche d'une source capable de saturer l'indétermination et la matérialisation lexicale ou non de la source à récupérer par le marqueur, que nous appellerons désormais « site » dans le couple « source-site » : [+/-LEX] ;

- le caractère unilatéral ou non du rapport entre la source et le site dans la construction de la référence [+/-UNI] ;

- la saturation par le marqueur et éventuellement sa source, d'une fonction au sein des prédications impliquées.

La combinaison de ces traits syntaxo-sémantiques aboutit à plusieurs types de rapports dont la concrétisation est plus ou moins bien attestée dans les divers stades de développement diachroniques, diastratiques ou diaphasiques de la langue. Nous décrirons infra les combinaisons possibles (§ 4-7).

\section{RAPPORT [+LEX] ET [+UNI] : CONFIGURATION " AVEC ANTÉCÉDENT "}

La configuration syntaxo-sémantique dans laquelle entrent les formes en quprésente dans ce cas-ci les trois traits suivants : (a) matérialisation lexicale d'une source, (b) saturation de fonction au sein des deux prédications (qualification de la source dans Préd 1 et site à charge fonctionnelle dans Préd 2) et (c) établissement d'une relation unilatérale entre la source et le site, comme en (5) (voir aussi les exemples (1a-c)) :

(5) Voilà le livre que tu m'avais demandé.

La proforme que renvoie à une source lexicale matérialisée, livre, qu'elle récupère anaphoriquement. La source et le site assument une fonction au sein de chacune des prédications et le rapport unilatéral source-site déclenche l'instauration d'une hiérarchisation syntaxique entre les prédications. Ce type de combinaison apparait pour toutes les formes, même s'il s'agit dans certains cas d'une variété plus informelle :

(6) À vous de voir si vous souhaitez garder le suspense pour le moment pour quand vous voudrez regarder la série. (tutusmelody.free.fr/site/mermaid_no_concerto/site/accueil)

(7) a. Si tu n'es pas content de la manière comment ce site est opéré, personne ne te retient [...]. (forum.palmattitude.org/index.php?showtopic)

b. [...] les parrains présentent les Ong candidates et font un rapport succinct sur la façon comment les candidats remplissent les [...]. (www.cercle.lu/article.php3) 
Ce qui caractérise les emplois de qu- dans la configuration [+LEX] - [+UNI], $c^{\prime}$ est que, sous l'effet de la présence de la source, elles tendent à perdre leur sens de base animé (8a), inanimé ( $8 b$ et 5$)$, lieu (8c), temps (6) et manière (7), pour reprendre le sens de leur source (antécédent) :

(8) a. Voilà le livre qui m'avait été demandé.

b. Il a vu l'homme que tu as dénoncé à la police.

c. Le moment où l'acteur fait son entrée est vraiment bien choisi. (Muller, $1996: 8)$

Les proformes, dans ces exemples, donnent la priorité à une fonction syntaxique : sujet \pm animé pour qui, objet \pm animé pour que, actant indirect ou circonstant pour où.

\section{RAPPORT [-LEX] ET [-UNI] : CONFIGURATION " DISCURSIVE "}

Les trois traits suivants caractérisent cette configuration syntaxo-sémantique : (a) non-matérialisation lexicale d'une source, (b) absence de saturation de fonction au sein des deux prédications et (c) non-instauration d'une relation unilatérale entre la source et le site : la relation [+/-UNI] cède ici la place à un mécanisme de renvoi déictique vers la prédication précédente, sans matérialisation d'une source lexicale précise. L'absence d'une source au sein de l'énoncé même entrave également la hiérarchisation syntaxique entre les prédications : la proforme en $q u$ - se limite à établir un rapport cohésif sans intégration syntaxique. La ponctuation souligne souvent cette non-intégration (9-10), même si la présence du morphème $c e$ explicite en quelque sorte un pointage vers une source propositionnelle antérieure (11). La plupart des marqueurs en $q u$ - entrent également dans cette configuration discursive :

(9) Il a refusé, d'où il résulte maintenant que nous sommes dans l'impasse. (Grevisse \& Goosse, 1986 : 1090)

(10) Le général Weygand prend acte de mes dispositions. Après quoi, il me parle de la bataille. (Grevisse \& Goosse, 1986 : 1090)

(11) a. Sous l'assaut, Venant ne broncha pas d'un cil. Ce qui plut infiniment à Didace. (Guèvrement, Le survenant, 1974)

b. Un son, disent-ils, [...], il vous faut le mesurer avec d'autant plus de précision : en hauteur, en densité, en timbre, en puissance etc. Ce à quoi l'ingénieur du son, habitué depuis longtemps à user de ses divers cadrans, leur répond que [...]. (Schaeffer, La musique concrète, 1967)

Dans les exemples (12) et (13), la source évoquée est encore plus indéterminée et la prédication introduite par le marqueur en $q u$-fonctionne comme une incise qui peut occuper plusieurs positions dans l'énoncé :

(12) Vos prisonniers, à ce que je vois, ont la permission de fumer. (Sandfeld, 1965: 110)

(13) Avouez que, pour ce qui est d'être bon, il était bon. (Sandfeld, 1965 : 111) 


\section{RAPPORT [+LEX] ET [-UNI] : CONFIGURATION " CORRÉLATIVE "}

Cette configuration syntaxo-sémantique, qui engendre des énoncés traditionnellement classés par les grammaires parmi les " corrélatives », présente les trois traits suivants : (a) matérialisation lexicale d'une source, (b) saturation de fonction au sein des deux prédications : le site - un relatif analytique ou non (par ex. (celui) qui, cf. infra § 8.2) - dans la première prédication, la source - un déictique se présentant souvent sous une forme composée (par ex. celui-ci) - dans la seconde prédication et (c) non-instauration d'une relation unilatérale entre la source et le site. Contrairement à la configuration de la relative postnominale (cf. type $[+\mathrm{LEX}]-[+\mathrm{UNI}]$ ), la matérialisation lexicale d'une source ne va pas de pair ici avec un rapport unilatéral source-site car la source elle-même est un déictique qui renvoie à la forme en qu- (et la construction qu'elle introduit); de plus, il y a généralement inversion du rapport source-site, le second apparaissant avant la première. La nature bilatérale du rapport source-site empêche la hiérarchisation entre les deux prédications.

Tous les marqueurs acceptent cette configuration, même s'ils apparaissent souvent sous forme analytique.

(14) Celui qui tentera d'expliquer en dix gros volumes [...], celui-ci entreprendra une besogne surhumaine. (Mounier, Traité du caractère, 1946)

(15) Ce qui a été dit de l'humanité, [...], comme cela se vérifie aujourd'hui pour la France! (Mauriac, Journal du temps de l'Occupation, 1952)

(16) Et tout ce que j'essaye de faire en vous, il faudrait que cela fût défait par ce garçon que je ne connais pas! (Montherlant, La ville dont le prince est un enfant, 1951)

(17) a. Où est Dieu, là nous serons avec Lui ! (Claudel, dans le TLFi)

b. Là où est la France, là est la patrie. (Gambetta, dans le Robert)

c. En général, partout où il se trouvait un monastère, là se formait un village. (Chateaubriand, Génie, 1803)

(18) Quand il s'agira de défendre vos intérêts matériels ou financiers, alors vous ne plaisanterez pas du tout. (Benzitoun, 2007b : 245)

(19) a. Comme le soleil chasse les ténèbres, ainsi la science chasse l'erreur. (www.mediadico.com/dictionnaire/definition/ainsi)

b. Tout comme il $\mathrm{n}^{\prime} \mathrm{y}$ a de poésie qu'autant que d'abord il y a des poèmes, de même il $\mathrm{n}^{\prime} \mathrm{y}$ a le cinéma qu'autant qu'il $\mathrm{y}$ a des films. (www.artcinema.org/spip.php?article23)

Le fonctionnement en diptyque inverse peut également être relevé. Ce qui est intéressant de noter ici, c'est la complémentarité (dans les exemples récoltés) avec le diptyque normal pour les formes en qui : alors que celui-ci se construit selon le schéma " marqueur analytique en qui - proforme source», le diptyque inverse préfère « proforme source - marqueur qui ».

(20) Tel est pris qui croyait prendre. (Wilmet, 2010 : § 672-2)

(21) a. [À propos d'histoires inventées] Mais celle-ci reste d'actualité, qui circulait à travers l'Europe [...]. (Gide, Ainsi soit-il, 1952) 
b. [...] mais ceux-là vinrent qui en avaient les moyens ou [...]. (Bazin, Vipère au poing, 1948)

(22) Mais ça ne m'apprend rien à moi tout ce que tu me racontes, dit Des Cigales. (Queneau, Loin de Rueil, 1944)

Pour ce qui est de l'exemple (22), il nous fait insensiblement glisser de la configuration [+LEX] - [-UNI] vers une configuration [-LEX] - [+UNI]. En effet, ça est ambigu : il est possible qu'il ne soit pas la source saturant ce que et qu'il fonctionne comme substitut au sein de Préd 1 d'une Préd 2 extraposée. Mais il pourrait tout aussi bien s'aligner sur le marqueur cela de l'exemple (15), qui tend à spécifier le parcours de toutes les variables possibles déclenché par ce qui dans Ce qui a été dit de l'humanité. La question se pose de savoir si l'énoncé (22), tout comme (15) d'ailleurs, implique cette opération de spécification ou de fléchage, i.e. s'il y a présence d'une source sous forme de ça ou si nous avons affaire à [-LEX] comme dans les cas qui seront décrits en 7.

\section{RAPPORT [-LEX] ET [+UNI] : CONFIGURATION " À ANTÉCÉDENT INCLUS "}

Cette dernière configuration syntaxo-sémantique, généralement appelée " relatif sans antécédent » ou "relatif libre », présente les traits suivants : (a) absence de matérialisation lexicale d'une source, (b) saturation de fonction au sein des deux prédications (le marqueur en $q u$ - exerce une fonction au sein de la seconde prédication et absorbe la source ainsi que sa charge fonctionnelle au sein de la première prédication), (c) établissement $d$ 'une relation unilatérale " interne » entre la source et le site. L'absence de lexicalisation de la source s'explique par le fait que celle-ci se trouve absorbée par le site, qui en arrive ainsi à assumer une fonction dans chacune des prédications, d'où un effet de hiérarchisation par co-saturation. De plus, la non-matérialisation de la source a pour conséquence de laisser vide la position qu'elle aurait occupée (23a, 24a), position qui peut cependant être remplie par une forme non-autonome marquant le genre et le nombre de la source non matérialisée (cf. § 8.2). Dans beaucoup de cas les deux options coexistent : «j'aime qui tu aimes » $v s$ " j'aime celui que tu aimes ». L'absorption de la source par le site ne change cependant rien au rapport sourcesite qui reste unilatéral. Les exemples en (23-24) confirment ce constat : le premier constituant-source attribue au second constituant-site et à la prédication qu'il introduit (i) le trait sémantique de base $(23 a-b)$, contrairement à la relative classique (23c), et (ii) la fonction dans la principale (24a-b) :

(23) a. Voila à qui / de quoi / où il parle.

b. Voilà [la personne à qui / la chose dont / l'endroit où] il parle.

c. C'est la chose qui a été résolue / que tu mªs donnée / à quoi tu fais référence.

(24) a. J'ai rencontré à qui tu as parlé hier.

b. J'ai rencontré [la personne] à qui tu as parlé hier. 
Comme le montrent les exemples (25-31), la position de $q u$ - $Q$ est variable dans l'énoncé, du moins pour ce qui est des marqueurs qui, que/quoi, où, quand. Nous n'avons pas trouvé d'occurrences de comme $Q$ antéposé avec saturation d'une fonction dans les deux prédications. Ce marqueur semble préférer la configuration corrélative, où s'établit un rapport ana-cataphorique avec la source (ainsi). De plus, théoriquement la fonction exercée par $q u$ - dans les deux prédications devrait pouvoir être identique ou distincte, mais en réalité, dans le cas de où, quand, comme, il y a toujours identité fonctionnelle dans les exemples récoltés, sauf dans des occurrences telles que (31) où qu- $Q$ exerce la fonction d'objet dans la première prédication :

(25) a. Qui critique est un traître, qui ne soutient pas ostensiblement la république est un suspect. (Camus, L'homme révolté, 1951)

b. [...] car je ne sais point respecter qui se trompe et [...]. (Saint-Exupéry, Citadelle, 1948)

(26) Advienne que pourra. (Wilmet, 2010 : § 678-a)

(27) a. Je me suis assis sur quoi tu t'es assis. (Muller, $1989: 22$ )

b. Il a remis au premier plan ce qui fait l'originalité profonde de son mouvement, par quoi il est si précieux à une réflexion sur la révolte, [...]. (Camus, L'homme révolté, 1951)

(28) a. Il n'est jamais où je le cherche, ce petit monsieur-là ! (Duhamel, La chronique des Pasquier, 1933-1945)

b. Où les chevaux d'Attila passaient, l'herbe ne poussait pas. (Muller, $1996: 13)$

(29) a. Je partirai quand tu partiras.

b. Quand nous déjeunions sur la terrasse, nous pouvions entendre chanter les oiseaux. (Muller, 1996 : 9)

(30) a. Les employés des divers bureaux m'accueillirent comme on accueille un sportif. (Nothomb, Stupeur et tremblement, 1999)

b. Je le regardai comme on regarde le Messie. (Nothomb, Stupeur et tremblement, 1999)

c. Enfin bon, tu fais comme tu veux. (Echenoz, Je m'en vais, 1999)

(31) a. C'était pas joli ça vous savez fallait voir ce qui s'est passeì laİ-bas on avait pas ouİ aller faire des besoins ni rien. (Benzitoun, 2007a : 7)

b. Pense à quand tu seras devenu médecin. (Muller, 1996 : 9)

c. J'aime aussi beaucoup quand il parle d'histoire naturelle. (Gide, Petit Robert)

Enfin, il nous reste à mentionner que les relatives "à antécédent inclus " acceptent l'extraposition et que la place laissée vide se trouve comblée par un pronom personnel ou par une autre trace, comme le déterminant possessif (cf. supra : la discussion des exemples 15 et 22).

(32) a. Celui qui gueulera le plus fort, il aura la médaille et la dragée du bon Jésus ! (Céline, TLFi)

b. Celui qui sera mon mari, je veux l'aimer. (Sandfeld, 1965 : 97)

c. Celui qui me gênera, j'aurai sa peau. (La Revue de France 9-12, $1927: 33$ )

(33) Je saurais le remercier princièrement, qui me rapporterait ce portefeuille. (Wilmet, $2007:$ § 206) 
C'est au niveau de l'élément de reprise que les relatives extraposées diffèrent formellement des " corrélatives ». Pourtant, l'écart sémantique entre les deux structures est ténu : dans le cas des " corrélatives », le morphème démonstratif constituera la source du morphème $q u$ - et déclenchera ainsi un rapport anacataphorique, alors que les éléments de reprise dans les exemples (32-33) ne constituent pas la source du $q u$ - mais reprennent la prédication extraposée dans son ensemble.

\section{PROPRIÉTÉS EXTERNES DES PROFORMES INDÉFINIES : BILAN}

La combinaison des propriétés externes des proformes indéfinies en $q u$ - donne lieu à quatre configurations déterminant le fonctionnement de celles-ci. Lorsque $q u$ - possède la propriété de matérialiser lexicalement une source [+LEX], il sera en mesure de saturer une fonction et éventuellement d'établir une relation unilatérale avec la source. Avec le trait [+UNI], il sera dit " relatif à antécédent ", avec le trait [-UNI] il entre dans une relation bilatérale avec sa source, ce qui caractérise le fonctionnement « corrélatif ». Dans les cas où il n'a pas la capacité de matérialiser une source ([-LEX]), il se peut qu'il renonce à la saturation fonctionnelle et à la création d'une relation source-site : il assumera le rôle de connecteur discursif. Mais il arrive aussi qu'il compense le trait [-LEX] en absorbant en quelque sorte une source qui reste indéterminée dans le contexte discursif ; il exercera alors une fonction dans les deux prédications et explicitera une relation unilatérale avec la source qu'il intègre en lui.

La variation dans les propriétés externes est à la base de certains glissements ou rapprochements entre emplois des marqueurs en $q u-$, comme les oscillations interprétatives entre les configurations " corrélative » et " relative à antécédent inclus » signalées précédemment. Le rapprochement possible est à situer, selon nous, dans la difficulté à réaliser simultanément deux traits syntaxo-sémantiques que la configuration "à antécédent inclus » est la seule à combiner : la cosaturation fonctionnelle et la non-lexicalisation de la source. Le fait de faire assumer par un seul marqueur l'exercice d'une fonction dans deux prédications semble difficile à maintenir. Dans la " corrélative », par contre, où la source reste également indéterminée, celle-ci est matérialisée, précisément de manière sous-spécifiée, par le démonstratif. La double construction avec des marqueurs distincts (marqueur en $q u$ - et démonstratif) permet de scinder la charge fonctionnelle dans les deux prédications et évite le cumul de [-LEX] et [+UNI] dans une seule forme.

D'autres phénomènes affectant le fonctionnement des proformes en $q u$ - révèlent l'instabilité des propriétés de la configuration "à antécédent inclus ", instabilité qui découle de la difficulté à combiner co-saturation et non-lexicalisation de la source. 


\subsection{Abandon de la co-saturation fonctionnelle pour assurer le rapport hiérarchique entre prédications}

La non-matérialisation lexicale de la source [-LEX] peut donner lieu à l'abandon de la co-saturation fonctionnelle par le marqueur en qu-, comme c'était déjà le cas dans la configuration discursive. Mais, contrairement à cette dernière, il existe des cas où l'abandon n'implique pas la suspension, pour qu-, de sa capacité de hiérarchisation syntaxique des prédications. Comme il n'est plus question de relation unilatérale " source-site ", ni de saturation fonctionnelle dans les deux prédications, le marqueur se limite à exercer une fonction dans la prédication régissante.

(34) a. Il a commencé à pleuvoir quand, en fin de soirée, il est sorti du cinéma.

b. Des pays comme l'Allemagne, la Suède ou le Danemark ont appuyé la résolution.

(35) a. Qui pourrait en détourner Lysandre, ce serait le plus sûr. (Corneille, $L a$ galerie du palais, v. 1609 ; cité par Muller, 2006 : 323)

« S'il pouvait en détourner Lysandre, ce serait le plus sûr »

b. Où son cœur contredisait ses principes, ses principes étaient d'avance vaincus. (Sandfeld, $1965: 119$ )

"Quand / si son cœur contredisait ses principes, ses principes étaient d'avance vaincus »

(36) Chaque Arménien perd un proche quand ce n'est pas toute sa famille. (Benzitoun, 2007b : 237)

"Chaque Armeìnien perd un proche si ce n'est pas toute sa famille »

(37) a. Comme elle était relativement fine au début, le brise-glace commença de s'y frayer un chemin frontalement. (Nothomb, Stupeur et tremblement, 1999)

«Étant donné qu'elle était relativement fine au début, le brise-glace [...] »

b. Un jour comme je tapotais pitoyablement sur la machine, je levai les yeux et je vis ma supérieure qui m'observait avec consternation. (Nothomb, Stupeur et tremblement, 1999)

«Un jour alors que je tapotais pitoyablement sur la machine, je levai les yeux $[\ldots] »$

La réduction au niveau des propriétés externes va souvent de pair avec une perte du sens sémantique de base, comme en témoignent les exemples (35-37). Mais le système peut essayer de compenser d'une autre manière la non-lexicalisation de la source pour maintenir la co-saturation fonctionnelle.

\subsection{Développement d'un marqueur analytique pour " relexicaliser " la source [+pseudoLEX]}

Nous avons déjà souligné que les marqueurs en qu- se réalisent tantôt sous forme simple, tantôt sous forme analytique. Avec cette dernière réalisation, la source " relexicalisée » facilite la double saturation fonctionnelle, d'où son apparition dans la configuration "relative à antécédent inclus ». Les énoncés (38a-b) et (39a-b) illustrent simultanément le fonctionnement du marqueur simple (qui en 38, quoi en 39a) et analytique (celui qui en 38 et ce dont en 39b). La forme 
analytique du marqueur en $q u$ - ne doit cependant pas être confondue avec la source démonstrative lexicalisée d'une configuration « relative avec antécédent » $(40 \mathrm{a}-\mathrm{b})$ :

(38) a. Qui n'a jamais donné un million à une femme ne les connaît pas, mais celui qui n'a jamais bouffé la dot de sa légitime ne les connaît pas non plus. (Cendrars, Bourlinguer, 1948)

b. Mais celui qui lit les poèmes [...] marquera d'une pierre blanche le jour où il peut entendre [...] les échos de la maison perdue. À qui sait écouter la maison du passé, n'est-elle pas une géométrie d'échos? (Bachelard, Poétique de l'espace, 1957)

(39) a. Voici de quoi ils ont parlé.

b. Il m'a communiqué ce dont ils ont parlé.

(40) a. Qu'ils chantent la passion, ceux-là dont la vie est accordée à la croix. (Sandfeld, 1965 : 100)

b. [...], tout cela, qui constituait une sorte d'organisme gigantesque, [...]. (Sandfeld, 1965 : 100)

L'oral informel a tendance à faire précéder le démonstratif d'un déterminant (dét dém qu-), ce qui confirme le fonctionnement comme marqueur analytique (41) :

(41) a. De ce que je comprends de ces lois, c'est qu'elles ne punissent pas les athées, mais les ceux qui osent quitter la religion d'état. (fr.wikipedia.org/wiki/Discussion:Discrimination_contre_les_athées)

b. Sujet: post de l'été pour les celles qui veulent maigrir doucement. (forum.doctissimo.fr/.../doucement-maigrir-veulent-sujet_174804_1.htm)

c. Si le celui qui détient le CG vend la voiture celui qui est marqué sur la facture à le droit d'en réclamer l'argent. (http:/ /auto.rtl.fr/article/)

d. C'est la celle qui a été la plus épargnée !! (www.commentcamarche.net/.../ affich-24740704)

Les formes en $q u$ - connaissent quasi toutes la double réalisation, simple ou analytique : voir les exemples (11a), (13), (14) et (15) pour qui, (11b), (12) et (16) pour que, (17b) et (17c) pour où et (42) pour comme. Dans leur réalisation analytique, elles sont souvent sujettes à des glissements sémantiques de la valeur de base vers des effets de sens dérivés (43):

(42) a. [...] paiement la facture correspondante ou la charge en cas de services facturables ainsi comme tout autre action qui implique par l'Utilisateur l'utilisation du site Web et les Services de ALCUCAR.COM. (www.alcucar.com/fr/aviso_legal.html)

b. Il est 9 heures du mat' j'ai déjà cassé tous mes serments, mais c'est ainsi comme ça que j'fonctionne jamais clean trop longtemps. (Starr, Jour de sortie, rapgenius.com/Joeystarr)

(43) a. Celui qui a dit ça, c'est parce qu'il n'est pas au courant des choses. "S'il a dit ça, c'est qu'il n'est pas au courant des choses »

b. Ce qui fait la force de l'Allemagne, c'est précisément parce que l'Allemagne n'a cessé de mettre au monde de futurs soldats. (Sandfeld, 1965 : 109)

« Si quelque chose fait la force de l'Allemagne, $c^{\prime}$ est le fait que l'Allemagne n'a cessé de mettre au monde de futurs soldats. » 
Dans le cas de ce que (44) et de là où (45), les glissements de sens peuvent être conséquents :

(44) a. Elle lui donne ce qu'elle a acheté. (+chose)

b. Elle est ce qu'elle est. (+propriété)

c. Cela vaut ce que ça vaut. (+quantité)

d. Ça durera ce que ça durera. (+temporel)

e. Quant aux Huit Heures, elles sont ici à la moto ce que Le Mans a été à sa plus belle époque à la voiture [...]. (Le Monde, 01.08.1989) (+identité)

(45) a. Et là où se marque le mieux son originalité, c'est dans les suppressions qu'il a faites. (Sandfeld, 1965 : 118) (+locatif)

b. Là où le français s'est séparé nettement des autres langues, c'est quand il a voulu faire un départ entre ces différentes formes. (Hadermann, 1993 : 170) (+temporel)

c. La bonne humeur et verve comique du Pédant, qui trouvait à rire là où d'autres eussent gémi comme veaux et pleuré comme vaches. (Gautier, TLFi) (+opposition)

Les marqueurs en $q u$-, au sein des formes composées, tendent donc à perdre leur propriété de représenter une source absorbée et leur valeur sémantique originelle en arrive à s'estomper (cf. 35-37 et 43-45). Cette double perte, de propriétés fonctionnelles et sémantiques, relève sans aucun doute d'une tendance à la grammaticalisation des marqueurs en $q u$ - (cf. entre autres Bat-Zeev Shyldkrot \& Kemmer 1988 ; Bertin, Bat-Zeev Shyldkrot \& Soutet 2013).

\section{CONCLUSION}

Nous aimerions revenir à un des questionnements posés par les éditeurs de ce volume : la corrélation constitue-t-elle un outil opérationnel pour appréhender les mécanismes de liaison de prédications en français moderne ? Notre réponse sera nuancée. Le schème "source-site » joue un rôle central dans le développement des configurations syntaxiques impliquant les formes en $q u$-, mais il se diversifie fortement dans la concrétisation de celles-ci jusqu'à présenter des constructions syntaxiques nettement différenciées. Il permet néanmoins de mieux appréhender les emplois des formes en $q u$-dans toute leur diversité.

Les propriétés externes qui découlent du schème " source-site » caractérisent les divers emplois des marqueurs en qu- étudiés ici : ceux-ci ont été définis comme des proformes indéfinies non spécifiées en recherche de spécification qui, selon la configuration dans laquelle ils s'insèrent, revêtissent les traits [+/-LEX], [+/-UNI] et saturent une fonction dans les prédications liées. D’après la sélection opérée par $q u$ - parmi ces traits, la proforme entrera dans des configurations différentes.

Par ailleurs, il peut se produire des glissements entre ces configurations. Ainsi, $q u$ - [-LEX] aura tendance à « re-matérialiser » la source à récupérer et à se déguiser ainsi, sous forme analytique, en $[+\mathrm{LEX}]$ pour se rapprocher tantôt 
de $q u$ - " relatif avec antécédent », tantôt de qu- « corrélatif ». De plus, en tant que [-LEX] - [+UNI], il est supposé exercer une double fonction dans chacune des deux prédications, difficulté qu'il contournera également avec la forme analytique au sein de laquelle il voit sa charge fonctionnelle distribuée sur deux éléments. Mais il arrive aussi qu'il renonce au trait [+UNI] pour ne garder que le fonctionnement de marqueur de rapports hiérarchiques entre prédications, fonctionnement qu'il peut même délaisser lorsqu'il devient marqueur discursif.

En nous inspirant des descriptions typologiques, nous avons défini de manière plus serrée la construction corrélative comme étant une construction constituée de deux prédications et caractérisée par la présence d'une proforme indéfinie dans une prédication et d'un démonstratif dans l'autre. Entre ces deux marqueurs s'établit un rapport ana-cataphorique, avec une interdépendance des prédications comme conséquence, et l'effet de sens qui est véhiculé est celui d'un parcours de variables accompagné d'une opération de fléchage ou d'identification. Même si le français n'est pas considéré comme appartenant à la famille des « langues corrélatives » au sens strict, nous y avons aussi relevé la productivité de la construction et nous oserions même avancer que cette productivité s'explique justement par le fait que le français dispose d'un riche paradigme de proformes en qu- qui, grâce à leur indéfinition et leur sous-spécification, peuvent être impliquées dans une construction corrélative.

L'exploitation de la notion de corrélation se justifie donc dans la description des mécanismes de liaison prédicationnelle des proformes en $q u$ - en français moderne à deux niveaux. Le schème " source-site » fonde d'abord les différentes configurations syntaxiques dans lesquelles les formes en $q u$ - peuvent s'insérer. Ensuite, la construction corrélative stricto sensu constitue une des configurations centrales dans lesquelles les proformes sont opérationnelles. Sur ce plan, elle est une configuration discursive comme le sont la relative avec antécédent ou la relative à antécédent inclus.

\section{Références bibliographiques}

[ARCHIVES DU MONDE] http://www.lemonde.fr/service/faq_625905.html

[FRANTEXT] http://www.frantext.fr/

[Petit Robert] Robert P., Rey A. \& Rey-Debove J. (1995), Nouveau petit Robert : dictionnaire alphabétique et analogique de la langue française, Paris : Le Robert.

[Robert] Robert P. \& ReY A. (1985), Dictionnaire alphabétique et analogique de la langue française, Paris : Le Robert.

[TLFI] http://atilf.atilf.fr/

Bat-ZeEv ShyLdKROt H. \& Kemmer S. (1988), "Le développement sémantique des conjonctions en français : quelques concepts généraux ", Revue Romane 23, 9-19.

Benzitoun C. (2007a), "Annotation morphosyntaxique. Où est le problème ", Cahiers du Cental 4, 1-16.

BENZIToun C. (2007b), "Description morphosyntaxique du mot quand en français contemporain ", Lingvisticae Investigationes 30 (2), 321-324. 
BENZITOUN C. (2008), "Qui est quand ? Essai d'analyse catégorielle ", Langue française 158, 129-143.

Bertin A., BAt-ZeEv ShyLdKRot H. \& Soutet O. (éds) (2013), Langages $\mathrm{n}^{\circ} 190$ : Subordonnants et subordination : frontières, chevauchements et cas limites, Paris : Larousse/Armand Colin.

CHERVEL A. (1977), ... et il fallut apprendre à écrire à tous les petits Français : histoire de la grammaire scolaire, Paris : Payot.

CoRminboeuf G. (2007), "Coordination, subordination, corrélation ou énonciation autonome ? ", Travaux neuchâtelois de linguistique 47, 177-194.

CReissels D. (2006), Syntaxe générale, une introduction typologique 2 : la phrase, Paris : Hermès-Lavoisier.

DEN DIKKEN M. (2005), “Comparative correlatives comparatively”, Linguistic Inquiry 36, 497-532.

DEULOFEU J. (2008), "Quel statut pour l'élément QUE en français contemporain ? ", Langue française 158, 29-52.

FRUYT M. (2005), "La corrélation en latin : définition et description ", in P. de Carvalho \& F. Lambert (éds), Structures parallèles et corrélatives en grec et en latin, Saint-Etienne : Presses de l'Université de Saint-Etienne, 17-44.

GREVISSE M. \& GoosSE A. $\left(1986^{12}, 1993^{13}\right)$, Le bon usage : grammaire française, Paris/Louvainla-Neuve : De Boeck-Duculot.

HADERMANN P. (1993), Étude morphosyntaxique du mot 'où', Bruxelles : Duculot.

Lefeuvre F. \& Pierrard M. (2008), "Les proformes indéfinies : indéfinition et prédication ", Langue française 158, 3-12.

LEFEUVRE F. \& RossaRI C. (2008), "Les degrés de grammaticalisation du groupe prép. + quoi anaphorique ", Langue française 158, 86-102.

LE GoFFIC P. (1993), Grammaire de la phrase française, Paris : Hachette Éducation.

LIPTÁK A. (2009), “The landscape of correlatives: An empirical and analytical survey”, in A. Lipták (éd.), Correlatives cross-linguistically, Amsterdam/Philadelphia: Benjamins, 1-46.

MeLLET S. (2013), "Proposition pour fonder énonciativement l'unité des phénomènes de corrélation ", in O. Inkova \& P. Hadermann (éds), La corrélation : aspects syntaxiques et sémantiques, Genève : Droz, 85-108.

MignON F. (2009), " Histoire du terme corrélation dans la grammaire française ", Langages 174, 13-24.

MULLER C. (1989), "Sur la syntaxe et la sémantique des relatives indépendantes et des interrogatives indirectes partielles ", Revue Romane 24 (1), 13-48.

MULLER C. (1996), "Quand où sert de quand", Zeitschrift für französische Sprache und Litteratur CVI, 6-21.

MULLER C. (2006), "Sur les propriétés des relatives ", Cahiers de grammaire 30, 319-337.

PierRaRd M. (1998), "Syntagme nominal et pronoms : la grammaticalisation des pronoms essentiels indéfinis ", in A. Englebert et al. (éds), La ligne claire. De la linguistique à la grammaire, Paris/Bruxelles : Duculot, 33-54.

PierRard M. (2005), "Les proformes indéfinies : connexion de prédications et subordination ", in F. Lambert \& H. Nølke (éds), La syntaxe au cœur de la grammaire, Rennes: Presses Universitaires de Rennes, 235-244.

PierRard M. (2013), "Comme et aussi que dans les comparatives: des marqueurs de subordination ?", Langages 190, 33-50.

SAEZ F. (2011), La scalarité de l'intégration syntaxique : étude syntaxique, sémantique et pragmatique de la proposition en 'quand', Thèse de I'Université Toulouse II-Le Mirail. 
SANDFELD K. (1965), Syntaxe du français contemporain : les propositions subordonnées, Genève : Droz.

SRIVASTAV V. (1991), "The syntax and semantics of correlatives", Natural Language and Linguistic Theory 9, 637-686.

WILMET M. (2007), Grammaire rénovée du français, Bruxelles : De Boeck-Larcier.

WILMET M. $\left(2010^{5}\right)$, Grammaire critique du français, Bruxelles : De Boeck-Duculot. 